ANNALES

POLONICI MATHEMATICI

$93.2(2008)$

\title{
A finite difference method for quasi-linear and nonlinear differential functional parabolic equations with Dirichlet's condition
}

\author{
by LuCJAN SAPa (Kraków)
}

\begin{abstract}
We deal with a finite difference method for a wide class of nonlinear, in particular strongly nonlinear or quasi-linear, second-order partial differential functional equations of parabolic type with Dirichlet's condition. The functional dependence is of the Volterra type and the right-hand sides of the equations satisfy nonlinear estimates of the generalized Perron type with respect to the functional variable. Under the assumptions adopted, quasi-linear equations are a special case of nonlinear equations. Quasi-linear equations are also treated separately. It is proved that our numerical methods are consistent, convergent and stable. Error estimates are given. The proofs are based on the comparison technique. Examples of physical applications and numerical experiments are presented.
\end{abstract}

1. Introduction. Let $D_{t}:=\partial / \partial t$ and $D_{i}:=\partial / \partial x_{i}, D_{i j}:=\partial^{2} / \partial x_{j} \partial x_{i}$ for $i, j=1, \ldots, n$, where $t \in \mathbb{R}, x=\left(x_{1}, \ldots, x_{n}\right) \in \mathbb{R}^{n}$. Put $D_{x}:=$ $\left(D_{1}, \ldots, D_{n}\right)$ and $D_{x}^{(2)}:=\left[D_{i j}\right]_{i, j=1}^{n}$. Let functions $f: \Delta \rightarrow \mathbb{R}$ and $\varphi:$ $E_{0} \cup \partial_{0} E \rightarrow \mathbb{R}$ be given (the relevant sets are defined in Section 2.1). Consider a nonlinear second-order partial differential functional equation of parabolic type of the form

$$
D_{t} u(t, x)=f\left(t, x, u, D_{x} u(t, x), D_{x}^{(2)} u(t, x)\right)
$$

with the initial condition and the boundary condition of the Dirichlet type

$$
u(t, x)=\varphi(t, x) \quad \text { on } E_{0} \cup \partial_{0} E .
$$

The aim of this paper is to give a finite difference method for finding an approximate solution of problem (1.1), (1.2). The equation may be nonlinear with respect to second derivatives. Such an equation is called strongly nonlinear. The functional dependence is of the Volterra type (e.g., delays or Volterra type integrals).

2000 Mathematics Subject Classification: 65M12, 65M15, 65M06, 35R10.

Key words and phrases: nonlinear and quasi-linear differential functional equations of parabolic type, finite difference methods, stability and convergence, nonlinear estimates of the generalized Perron type. 
Partial differential equations of parabolic type give mathematical models of nonstationary processes of heat exchange or mass transport. Some complicated kinds of these phenomena involve equations with a functional term. Differential difference equations (e.g., with time or spatial delays) describe fast heat changes in nuclear reactors, while differential integral equations are used for integral heat sources in an anisotropic medium. Both can be connected with our equation.

In this paper, we construct an explicit finite difference functional scheme. It is proved that, under suitable assumptions on the reaction function $f$ and steps of a mesh, the method is consistent, convergent and stable. An error estimate of the approximate solution is given. The proof is based on the comparison technique with the use of a difference functional inequality proved by Z. Kamont [10] (see also [8]). These results in particular cover a quasi-linear differential functional equation of the form

$$
D_{t} u(t, x)=\sum_{i, j=1}^{n} a_{i j}(t, x, u) D_{i j} u(t, x)+F\left(t, x, u, D_{x} u(t, x)\right),
$$

where $a_{i j}: \Delta^{A} \rightarrow \mathbb{R}$ and $F: \Delta^{F} \rightarrow \mathbb{R}, i, j=1, \ldots, n$, are given functions (see Section 2.1). But for such an equation it is assumed that all of the coefficients $a_{i j}$ are of the same sign. To omit this condition, another scheme is also studied. These results can be extended to weakly coupled systems. At the end of the paper, we present numerical examples.

It follows from the convergence of the numerical methods that the differential functional problems considered have at most one classical solution. Theorems on the existence and uniqueness of such solutions for some special parabolic differential functional equations can be found in [3], [4], [7], [26] and the references therein.

Similar general strongly nonlinear parabolic difference functional numerical problems have been studied by Z. Kamont, H. Leszczyński, M. Malec, Cz. Mączka, W. Voigt and M. Rosati [8], [10], [13], [14], [15] and others. In those papers, the Lipschitz or Perron conditions are assumed. In our paper, we generalize the Perron estimate, multiplying a function $\sigma$ by some nondecreasing function $\varrho$ (see assumption $\left(F_{4}\right)$ in Section 3 ). This considerably extends the class of problems which are solvable with the method described. Under the assumptions adopted, our nonlinear equation includes as special cases the quasi-linear equation (1.3) and a strongly nonlinear equation with a quasi-linear term. Neither of these cases appears in the cited papers. This result is new, even for equations without a functional term (cf. [11]-[17]). Moreover, unlike some cited papers, we do not assume differentiability of $f$ with respect to the variables $p$ and $q$ (see Section 3). 
The results concerning numerical methods, differential functional and difference functional inequalities or the uniqueness theory, appearing in the papers of P. Besala and G. Paszek [1], [2], Z. Kamont and H. Leszczyński [8], [10], C. V. Pao [18]-[20], R. Redheffer and W. Walter [21], J. Szarski [22]-[24] and numerous others, do not apply to nonlinear equations and quasi-linear equations with general functional dependence as in our paper.

A finite difference method for quasi-linear parabolic differential functional equations (with functional dependence of the Hale type) with Dirichlet's condition has been considered by R. Ciarski [6]. In our paper, in contrast to [6], we do not assume differentiability of $F$ with respect to the variable $p$ (see Section 6.2). The functional term may be approximated by step functions or spline polynomials, while in [6] by spline polynomials only. Numerical approximation for such quasi-linear equations with Neumann's condition is treated in [5].

\section{Notation and definitions}

2.1. Sets and function spaces. Let $T>0, \delta=\left(\delta_{1}, \ldots, \delta_{n}\right), \tau_{0} \geq 0$, $\tau=\left(\tau_{1}, \ldots, \tau_{n}\right)$, where $\delta_{i}>0, \tau_{i} \geq 0$ for $i=1, \ldots, n$, be given real numbers. Define

$$
\begin{aligned}
E & :=[0, T] \times(-\delta, \delta) \subset \mathbb{R}^{1+n}, \\
E_{0} & :=\left[-\tau_{0}, 0\right] \times[-\delta-\tau, \delta+\tau] \subset \mathbb{R}^{1+n}, \\
\partial_{0} E & :=[0, T] \times([-\delta-\tau, \delta+\tau] \backslash(-\delta, \delta)) \subset \mathbb{R}^{1+n} .
\end{aligned}
$$

Let, moreover,

$$
\Omega:=E \cup E_{0} \cup \partial_{0} E, \quad \Omega_{t}:=\{(\widetilde{t}, x) \in \Omega: \widetilde{t} \leq t\} .
$$

A mapping $u: \Omega \rightarrow \mathbb{R}$ of class $C^{1,2}$ will be called regular on $\Omega$. We briefly write $u \in C^{1,2}(\Omega, \mathbb{R})$.

The set

$$
\begin{aligned}
& B(\Omega, \mathbb{R}):=\{z: \Omega \rightarrow \mathbb{R} \mid \sup \{|z(t, x)|:(t, x) \in \Omega\}<\infty, \\
& \exists k \in \mathbb{N} \exists \Omega_{1}, \ldots, \Omega_{k} \exists a^{(1)}, \ldots, a^{(k)}, a \in \mathbb{R} \exists b^{(1)}, \ldots, b^{(k)}, b \in \mathbb{R}^{n}: \\
& \Omega_{i}=\Omega \cap\left(\left[a^{(i)}, a^{(i)}+a\right) \times\left[b^{(i)}, b^{(i)}+b\right)\right), \\
&\left.\Omega=\bigcup_{i=1}^{k} \Omega_{i}, \Omega_{i} \cap \Omega_{j}=\emptyset \text { for } i \neq j,\left.z\right|_{\Omega_{i}} \in C\left(\Omega_{i}, \mathbb{R}\right), i, j=1, \ldots, k\right\}
\end{aligned}
$$

is the set of Lebesgue-measurable functions, bounded and piecewise continuous on $\Omega$. For a fixed $t \in[0, T]$,

$$
\|z\|(t):=\sup \left\{|z(\widetilde{t}, x)|:(\widetilde{t}, x) \in \Omega_{t}\right\}
$$

is a seminorm in the space $B(\Omega, \mathbb{R})$, where $z \in B(\Omega, \mathbb{R})$. 
Let $M_{n \times n}$ denote the class of all $n \times n$ real matrices. Define

$$
\begin{aligned}
\Delta & :=E \times B(\Omega, \mathbb{R}) \times \mathbb{R}^{n} \times M_{n \times n}, \\
\Delta_{1} & :=E \times B^{2}(\Omega, \mathbb{R}) \times \mathbb{R}^{2 n} \times M_{n \times n}^{2}, \\
\Delta^{F} & :=E \times B(\Omega, \mathbb{R}) \times \mathbb{R}^{n}, \quad \Delta_{1}^{F}:=E \times B^{2}(\Omega, \mathbb{R}) \times \mathbb{R}^{2 n}, \\
\Delta^{A} & :=E \times B(\Omega, \mathbb{R}) .
\end{aligned}
$$

The maximum norms in $\mathbb{R}^{n}$ and $M_{n \times n}$ are denoted by $\|\cdot\|$, and the maximum norm in $C(\Omega, \mathbb{R})$ by $\|\cdot\|_{\Omega}$. Similarly, $\|\cdot\|_{\Omega}$ stands for the supremum norm in $B(\Omega, \mathbb{R})$.

2.2. Discretization, difference, step and interpolation operators. We use vectorial inequalities to mean that the same inequalities hold between the corresponding components. We write $x \diamond y=\left(x_{1} y_{1}, \ldots, x_{n} y_{n}\right)$ for $x=$ $\left(x_{1}, \ldots, x_{n}\right), y=\left(y_{1}, \ldots, y_{n}\right) \in \mathbb{R}^{n}$. Define a mesh on the set $\Omega$ in the following way. Let $\left(h_{0}, h^{\prime}\right)=h, h^{\prime}=\left(h_{1}, \ldots, h_{n}\right)$, stand for steps of the mesh. Denote by $H$ the set of all $h$ such that there exist $N_{0} \in \mathbb{Z}$ and $N=\left(N_{1}, \ldots, N_{n}\right) \in \mathbb{N}^{n}$ with the properties: $N_{0} h_{0}=\tau_{0}, N \diamond h^{\prime}=\delta+\tau$. Obviously, $H \neq \emptyset$ and there are $K_{0} \in \mathbb{N}$ and $K=\left(K_{1}, \ldots, K_{n}\right) \in \mathbb{Z}^{n}$ such that $K_{0} h_{0} \leq T<\left(K_{0}+1\right) h_{0}, K \diamond h^{\prime}<\delta \leq(K+1) \diamond h^{\prime}$. For $h \in H$ and $(\mu, m) \in \mathbb{Z}^{1+n}, m=\left(m_{1}, \ldots, m_{n}\right)$, we define nodal points $\left(t^{(\mu)}, x^{(m)}\right)$, $x^{(m)}=\left(x_{1}^{\left(m_{1}\right)}, \ldots, x_{n}^{\left(m_{n}\right)}\right)$ in the following way:

$$
t^{(\mu)}:=\mu h_{0}, \quad x^{(m)}:=m \diamond h^{\prime} .
$$

For $h \in H$, we put

$$
R_{h}^{1+n}:=\left\{\left(t^{(\mu)}, x^{(m)}\right):(\mu, m) \in \mathbb{Z}^{1+n}\right\} .
$$

Next, we define the discrete sets

$$
\begin{array}{rlrl}
E_{h} & :=E \cap R_{h}^{1+n}, & & \\
E_{0 . h} & :=E_{0} \cap R_{h}^{1+n}, & \partial_{0} E_{h}:=\partial_{0} E \cap R_{h}^{1+n}, \\
\Omega_{h} & :=E_{h} \cup E_{0 . h} \cup \partial_{0} E_{h}, \quad \Omega_{t . h}:=\Omega_{t} \cap R_{h}^{1+n} .
\end{array}
$$

Let, moreover,

$$
\begin{gathered}
E_{h}^{+}:=\left\{\left(t^{(\mu)}, x^{(m)}\right) \in E_{h}: 0 \leq \mu \leq K_{0}-1\right\}, \\
I_{h}:=\left\{t^{(\mu)}: 0 \leq \mu \leq K_{0}\right\}, \quad I_{h}^{+}:=\left\{t^{(\mu)}: 0 \leq \mu \leq K_{0}-1\right\} .
\end{gathered}
$$

For a mesh function $a: \Omega_{h} \supset A_{h} \rightarrow \mathbb{R}$ and a point $\left(t^{(\mu)}, x^{(m)}\right) \in A_{h}$, we put $a^{(\mu, m)}:=a\left(t^{(\mu)}, x^{(m)}\right)$. We denote the space of all such functions by $F\left(A_{h}, \mathbb{R}\right)$ and call it the space of mesh functions. In $F\left(A_{h}, \mathbb{R}\right)$, we introduce the maximum norm

$$
\|a\|_{A_{h}}:=\max \left\{\left|a^{(\mu, m)}\right|:\left(t^{(\mu)}, x^{(m)}\right) \in A_{h}\right\},
$$

where $a \in F\left(A_{h}, \mathbb{R}\right)$. 
For a fixed $\mu \in\left\{0,1, \ldots, K_{0}\right\}$,

$$
\|a\|_{h}(\mu):=\max \left\{\left|a^{(\widetilde{\mu}, m)}\right|:\left(t^{(\widetilde{\mu})}, x^{(m)}\right) \in \Omega_{t^{\mu} . h}\right\}
$$

is a seminorm in the space $F\left(\Omega_{h}, \mathbb{R}\right)$, where $a \in F\left(\Omega_{h}, \mathbb{R}\right)$.

For a function $a: I_{h} \supset A_{h} \rightarrow \mathbb{R}_{+}$, we put $a^{(\mu)}:=a\left(t^{(\mu)}\right), t^{(\mu)} \in A_{h}$, where $\mathbb{R}_{+}:=[0,+\infty)$.

Write

$$
\Gamma:=\{(i, j): 1 \leq i, j \leq n, i \neq j\}
$$

and suppose that $\Gamma_{+}, \Gamma_{-} \subset \Gamma$ are such that $\Gamma_{+} \cup \Gamma_{-}=\Gamma, \Gamma_{+} \cap \Gamma_{-}=\emptyset$ (in particular, it may happen that $\Gamma_{+}=\emptyset$ or $\left.\Gamma_{-}=\emptyset\right)$. We assume that $(i, j) \in$ $\Gamma_{+}$when $(j, i) \in \Gamma_{+}$, and $(i, j) \in \Gamma_{-}$when $(j, i) \in \Gamma_{-}$. Let $a \in F\left(\Omega_{h}, \mathbb{R}\right)$ and $\left(t^{(\mu)}, x^{(m)}\right) \in E_{h}^{+}$. Set

$$
\begin{aligned}
\delta_{i}^{+} a^{(\mu, m)} & :=\frac{1}{h_{i}}\left[a^{\left(\mu, m+e_{i}\right)}-a^{(\mu, m)}\right], \\
\delta_{i}^{-} a^{(\mu, m)} & :=\frac{1}{h_{i}}\left[a^{(\mu, m)}-a^{\left(\mu, m-e_{i}\right)}\right],
\end{aligned}
$$

where $e_{i}=(0, \ldots, 0,1,0, \ldots, 0)$ with 1 in the $i$ th entry, $i=1, \ldots, n$. We apply the difference quotients $\delta_{0}, \delta=\left(\delta_{1}, \ldots, \delta_{n}\right), \delta^{(2)}=\left[\delta_{i j}\right]_{i, j=1}^{n}$ given by

$$
\begin{aligned}
\delta_{0} a^{(\mu, m)} & :=\frac{1}{h_{0}}\left[a^{(\mu+1, m)}-a^{(\mu, m)}\right], & & \\
\delta_{i} a^{(\mu, m)} & :=\frac{1}{2}\left[\delta_{i}^{+} a^{(\mu, m)}+\delta_{i}^{-} a^{(\mu, m)}\right] & & \text { for } i=1, \ldots, n, \\
\delta_{i i} a^{(\mu, m)} & :=\delta_{i}^{+} \delta_{i}^{-} a^{(\mu, m)} & & \text { for } i=1, \ldots, n, \\
\delta_{i j} a^{(\mu, m)} & :=\frac{1}{2}\left[\delta_{i}^{+} \delta_{j}^{-} a^{(\mu, m)}+\delta_{i}^{-} \delta_{j}^{+} a^{(\mu, m)}\right] & & \text { for }(i, j) \in \Gamma_{-}, \\
\delta_{i j} a^{(\mu, m)} & :=\frac{1}{2}\left[\delta_{i}^{+} \delta_{j}^{+} a^{(\mu, m)}+\delta_{i}^{-} \delta_{j}^{-} a^{(\mu, m)}\right] & & \text { for }(i, j) \in \Gamma_{+} .
\end{aligned}
$$

We use these operators to approximate derivatives in equations (1.1) and (1.3).

Define the step operator $S_{h}: F\left(\Omega_{h}, \mathbb{R}\right) \rightarrow B(\Omega, \mathbb{R})$ by the formula

$$
S_{h}[a](t, x):=\sum_{\left(t^{(\mu)}, x^{(m)}\right) \in \Omega_{h}} \chi_{(\mu, m)}(t, x) a^{(\mu, m)} \quad \text { for }(t, x) \in \Omega,
$$

where $a \in F\left(\Omega_{h}, \mathbb{R}\right)$ and

$$
\begin{gathered}
\chi_{(\mu, m)}(t, x):= \begin{cases}1 & \text { for }(t, x) \in J_{(\mu, m)}, \\
0 & \text { for }(t, x) \in \Omega \backslash J_{(\mu, m)},\end{cases} \\
J_{(\mu, m)}:=\left\{(t, x) \in \Omega: t^{(\mu)} \leq t<t^{(\mu+1)}, x^{(m)} \leq x<x^{(m+1)}\right\},
\end{gathered}
$$

where $m+1=\left(m_{1}+1, \ldots, m_{n}+1\right)(\mathrm{cf}$. [13]). 
Finally, define the interpolation operator $T_{h}: F\left(\Omega_{h}, \mathbb{R}\right) \rightarrow C(\Omega, \mathbb{R})$ in the following way. Put

$$
S_{+}:=\left\{s=\left(s_{1}, \ldots, s_{n}\right): s_{i} \in\{0,1\} \text { for } 1 \leq i \leq n\right\} .
$$

Let $a \in F\left(\Omega_{h}, \mathbb{R}\right)$ and $(t, x) \in \Omega$. There exists $\left(t^{(\mu)}, x^{(m)}\right) \in \Omega_{h}$ such that $\left(t^{(\mu+1)}, x^{(m+1)}\right) \in \Omega_{h}$ and $t^{(\mu)} \leq t \leq t^{(\mu+1)}, x^{(m)} \leq x \leq x^{(m+1)}$. Write

$$
\begin{aligned}
T_{h}[a](t, x) & \left(1-\frac{t-t^{(\mu)}}{h_{0}}\right) \sum_{s \in S_{+}} a^{(\mu, m+s)}\left(\frac{x-x^{(m)}}{h^{\prime}}\right)^{s}\left(1-\frac{x-x^{(m)}}{h^{\prime}}\right)^{1-s} \\
& +\frac{t-t^{(\mu)}}{h_{0}} \sum_{s \in S_{+}} a^{(\mu+1, m+s)}\left(\frac{x-x^{(m)}}{h^{\prime}}\right)^{s}\left(1-\frac{x-x^{(m)}}{h^{\prime}}\right)^{1-s},
\end{aligned}
$$

where

$$
\begin{aligned}
\left(\frac{x-x^{(m)}}{h^{\prime}}\right)^{s} & :=\prod_{i=1}^{n}\left(\frac{x_{i}-x_{i}^{\left(m_{i}\right)}}{h_{i}}\right)^{s_{i}}, \\
\left(1-\frac{x-x^{(m)}}{h^{\prime}}\right)^{1-s} & :=\prod_{i=1}^{n}\left(1-\frac{x_{i}-x_{i}^{\left(m_{i}\right)}}{h_{i}}\right)^{1-s_{i}} .
\end{aligned}
$$

We adopt the convention that $0^{0}=1$ (cf. [9]).

We apply the operators $S_{h}$ and $T_{h}$ to approximate the functional term in equations (1.1) and (1.3).

3. Differential functional problem. We need the following assumptions on the functions $f, \varphi$ and regularity of a solution $u$ of (1.1), (1.2).

Assumption $F[f, u]$

$\left(F_{1}\right)$ The function $f$ is continuous on $\Delta$.

$\left(F_{2}\right)$ There exist functions $\alpha=\left(\alpha_{1}, \ldots, \alpha_{n}\right), \beta=\left[\beta_{i j}\right]_{i, j=1}^{n}$ with $\alpha_{i}, \beta_{i j}$ : $\Delta_{1} \rightarrow \mathbb{R}$ such that for any $(t, x, z, p, q),(t, x, z, \bar{p}, \bar{q}) \in \Delta$,

$$
\begin{aligned}
f(t, x, z, p, q)-f(t, x, z, \bar{p}, \bar{q}) & \\
& =\sum_{i=1}^{n} \alpha_{i}(P)\left(p_{i}-\bar{p}_{i}\right)+\sum_{i, j=1}^{n} \beta_{i j}(P)\left(q_{i j}-\bar{q}_{i j}\right),
\end{aligned}
$$

where $P=(t, x, z, z, p, \bar{p}, q, \bar{q}) \in \Delta_{1}$.

$\left(F_{3}\right)$ The matrix $\beta$ is symmetric and

$$
\begin{array}{ll}
\beta_{i j}(P) \geq 0 & \text { for }(i, j) \in \Gamma_{+}, \\
\beta_{i j}(P) \leq 0 & \text { for }(i, j) \in \Gamma_{-},
\end{array}
$$

at each $P \in \Delta_{1}$.

$\left(F_{4}\right)$ There are functions $\sigma:[0, T] \times \mathbb{R}_{+} \rightarrow \mathbb{R}_{+}, \varrho: \mathbb{R}_{+} \rightarrow \mathbb{R}_{+}$such that: 
(1) $\sigma$ is continuous and nondecreasing with respect to both variables; moreover, $\sigma(t, 0)=0$ for $t \in[0, T]$;

(2) $\varrho$ is nondecreasing;

(3) for each $c \geq 0$ and $\varepsilon, \varepsilon_{0}>0$, the maximum solution of the Cauchy problem

$$
\omega^{\prime}(t)=c \sigma(t, \omega(t))+\varepsilon, \quad \omega(0)=\varepsilon_{0},
$$

is defined on $[0, T]$ and the function $\widetilde{\omega}(t)=0$ for $t \in[0, T]$ is the maximum solution of problem (3.2) for each $c \geq 0$ and $\varepsilon, \varepsilon_{0}=0$

(4) the generalized Perron type estimate

$$
|f(t, x, z, p, q)-f(t, x, \bar{z}, p, q)| \leq \varrho(\|q\|) \sigma(t,\|z-\bar{z}\|(t))
$$

holds on $\Delta$.

$\left(F_{5}\right)$ The function $u \in C^{1,2}(\Omega, \mathbb{R})$ is a regular solution of (1.1), (1.2).

Remark 3.1. Assumptions (1) and (4) in $\left(F_{4}\right)$ imply that the functional $f$ is of the Volterra type. That is, if $t \in[0, T]$ and $z, \bar{z} \in B(\Omega, \mathbb{R}), z(\widetilde{t}, x)=$ $\bar{z}(\widetilde{t}, x)$ for $(\widetilde{t}, x) \in \Omega_{t}$, then $f(t, x, z, p, q)=f(t, x, \bar{z}, p, q)$ for $x \in(-\delta, \delta)$, $p \in \mathbb{R}^{n}, q \in \mathbb{R}^{n^{2}}$.

REMARK 3.2. If the reaction function $f$ is differentiable, then $\alpha, \beta$ in assumption $\left(F_{2}\right)$ involve derivatives of $f$.

4. Finite difference functional scheme. We define a finite difference functional scheme which will be applied to approximate a solution of the differential functional problem (1.1), (1.2). The functional term will be approximated by the step operator $S_{h}$. In Section 6.3, we show that it can be replaced by the interpolation operator $T_{h}$.

DeFinition 4.1. The finite difference functional scheme for the differential functional problem (1.1), (1.2) is the system of algebraic equations

$$
\left\{\begin{array}{l}
\delta_{0} a^{(\mu, m)}=f\left(t^{(\mu)}, x^{(m)}, S_{h}[a], \delta a^{(\mu, m)}, \delta^{(2)} a^{(\mu, m)}\right), \\
a^{(\mu, m)}=\varphi_{h}^{(\mu, m)} \text { on } E_{0 . h} \cup \partial_{0} E_{h},
\end{array}\right.
$$

where $\varphi_{h} \in F\left(E_{0 . h} \cup \partial_{0} E_{h}, \mathbb{R}\right)$ is a given function and $a \in F\left(\Omega_{h}, \mathbb{R}\right)$.

We shall use the following assumptions on the steps $h$ of the mesh $\Omega_{h}$.

Assumption $S[h]$

$\left(S_{1}\right)$ The steps $h=\left(h_{0}, h^{\prime}\right) \in H$ are such that

$$
1-2 h_{0} \sum_{i=1}^{n} \frac{1}{h_{i}^{2}} \beta_{i i}(P)+h_{0} \sum_{(i, j) \in \Gamma} \frac{1}{h_{i} h_{j}}\left|\beta_{i j}(P)\right| \geq 0,
$$




$$
-\frac{h_{i}}{2}\left|\alpha_{i}(P)\right|+\beta_{i i}(P)-h_{i} \sum_{\substack{j \neq i \\ j=1}}^{n} \frac{1}{h_{j}}\left|\beta_{i j}(P)\right| \geq 0
$$

at each $P \in \Delta_{1}, i=1, \ldots, n$ (see Assumption $F[f, u]$ ).

$\left(S_{2}\right)$ There is $c_{0}>0$ such that $h_{i} h_{j}^{-1} \leq c_{0}$ for $i, j=1, \ldots, n$.

REMARK 4.1. If Assumption $F[f, u]$ holds and there is a step $h \in H$ satisfying Assumption $S[h]$, then there exists a sequence of steps $h \in H$ which satisfy Assumption $S[h]$ and $h \rightarrow 0$.

5. Stability of difference functional equations. We now present a theorem due to Z. Kamont [10] (see also [8]). It will be applied in the proofs of convergence of the difference methods in Section 6 .

Suppose that an operator $F_{h}: E_{h}^{+} \times F\left(\Omega_{h}, \mathbb{R}\right) \rightarrow \mathbb{R}$ is given. For $\left(t^{(\mu)}, x^{(m)}, a\right) \in E_{h}^{+} \times F\left(\Omega_{h}, \mathbb{R}\right)$, we write $F_{h}[a]^{(\mu, m)}:=F_{h}\left(t^{(\mu)}, x^{(m)}, a\right)$. Given $\varphi_{h} \in F\left(E_{0 . h} \cup \partial_{0} E_{h}, \mathbb{R}\right)$, we consider the difference functional equation

$$
a^{(\mu+1, m)}=F_{h}[a]^{(\mu, m)}
$$

with the initial-boundary condition

$$
a^{(\mu, m)}=\varphi_{h}^{(\mu, m)} \quad \text { on } E_{0 . h} \cup \partial_{0} E_{h} .
$$

If $F_{h}$ satisfies the Volterra condition (see Remark 3.1), then there exists exactly one solution $v \in F\left(\Omega_{h}, \mathbb{R}\right)$ of $(5.1),(5.2)$. Note that the Volterra condition states that the value of $F_{h}$ at $\left(t^{(\mu)}, x^{(m)}, a\right)$ depends on $\left(t^{(\mu)}, x^{(m)}\right)$ and the restriction of the function $a$ to the set $\Omega_{t^{\mu}} . h$ only.

Let $Y_{h} \subset F\left(\Omega_{h}, \mathbb{R}\right)$ be a fixed subset. Suppose that a function $w \in Y_{h}$, a function $\gamma: I_{h}^{+} \rightarrow \mathbb{R}_{+}$and $\alpha_{0} \in \mathbb{R}_{+}$satisfy the conditions

$$
\begin{aligned}
\left|a^{(\mu+1, m)}-F_{h}[a]^{(\mu, m)}\right| & \leq \gamma^{(\mu)} & & \text { on } E_{h}^{+}, \\
\left|a^{(\mu, m)}-\varphi_{h}^{(\mu, m)}\right| & \leq \alpha_{0} & & \text { on } E_{0 . h} \cup \partial_{0} E_{h} .
\end{aligned}
$$

The function $w$ satisfying the above relations is considered an approximate solution of (5.1), (5.2).

The theorem below gives an estimate of the difference between the exact and approximate solutions of (5.1), (5.2).

TheOREM 5.1. Suppose that

(1) $F_{h}$ is of the Volterra type, $h \in H$,

(2) $\sigma_{h}: I_{h}^{+} \times \mathbb{R}_{+} \rightarrow \mathbb{R}_{+}$is nondecreasing with respect to the second variable and

$$
\begin{aligned}
& \left|F_{h}[a]^{(\mu, m)}-F_{h}[\bar{a}]^{(\mu, m)}\right| \leq \sigma_{h}\left(t^{(\mu)},\|a-\bar{a}\|_{h}(\mu)\right) \\
& \text { for }\left(t^{(\mu)}, x^{(m)}\right) \in E_{h}^{+}, a \in F\left(\Omega_{h}, \mathbb{R}\right), \bar{a} \in Y_{h}, \\
& \text { (3) } v \in F\left(\Omega_{h}, \mathbb{R}\right) \text { is the solution of problem }(5.1),(5.2),
\end{aligned}
$$


(4) $w \in Y_{h}$ and there are $\gamma: I_{h}^{+} \rightarrow \mathbb{R}_{+}, \alpha_{0} \in \mathbb{R}_{+}$such that (5.3) and (5.4) hold,

(5) $\beta: I_{h} \rightarrow \mathbb{R}_{+}$is nondecreasing and satisfies the recurrent inequality

$$
\beta^{(\mu+1)} \geq \sigma_{h}\left(t^{(\mu)}, \beta^{(\mu)}\right)+\gamma^{(\mu)}, \quad \mu=0, \ldots, K_{0}-1,
$$

and $\beta^{(0)} \geq \alpha_{0}$.

Then

$$
\|w-v\|_{h}(\mu) \leq \beta^{(\mu)}, \quad \mu=0, \ldots, K_{0} .
$$

REMARK 5.1. Let the assumptions of Theorem 5.1 be satisfied with

$$
\sigma_{h}(t, y):=\left(1+L h_{0}\right) y, \quad(t, y) \in I_{h}^{+} \times \mathbb{R}_{+},
$$

where $L \geq 0$ and there is $\widetilde{\gamma} \in \mathbb{R}_{+}$such that $\gamma^{(\mu)} \leq h_{0} \widetilde{\gamma}, \mu=0, \ldots, K_{0}-1$. Then

(i) if $L>0$, then

$$
\begin{aligned}
\|w-v\|_{h}(\mu) & \leq\left(1+L h_{0}\right)^{\mu} \alpha_{0}+\widetilde{\gamma} \frac{\left(1+L h_{0}\right)^{\mu}-1}{L} \\
& \leq \exp (L T) \alpha_{0}+\widetilde{\gamma} \frac{\exp (L T)-1}{L}
\end{aligned}
$$

for $\mu=0, \ldots, K_{0}$;

(ii) if $L=0$, then

$$
\|w-v\|_{h}(\mu) \leq \alpha_{0}+\mu h_{0} \widetilde{\gamma} \leq \alpha_{0}+T \widetilde{\gamma}
$$

for $\mu=0, \ldots, K_{0}$.

Assumption (2) in Theorem 5.1 states that $F_{h}$ with $\sigma_{h}$ defined above satisfies the Lipschitz condition with respect to the functional variable with Lipschitz constant $1+L h_{0}$. This remark is important in applications.

\section{Theoretical study of the scheme}

6.1. Convergence of the difference method. We now turn to the main problem of this paper, convergence of the difference method (4.1).

Let $U \in F\left(\Omega_{h}, \mathbb{R}\right)$ be the restriction of a regular solution $u \in C^{1,2}(\Omega, \mathbb{R})$ of (1.1), (1.2) to the mesh $\Omega_{h}$ and let $v \in F\left(\Omega_{h}, \mathbb{R}\right)$ be the solution of the finite difference functional scheme (4.1).

Definition 6.1. The difference method (4.1) is uniformly convergent if

$$
\lim _{h \rightarrow 0}\|r\|_{\Omega_{h}}=0,
$$

where $r:=U-v \in F\left(\Omega_{h}, \mathbb{R}\right)$ is the error of the method.

Theorem 6.1. Let Assumptions $F[f, u]$ and $S[h]$ hold and suppose that there is a function $\alpha_{0}: H \rightarrow \mathbb{R}_{+}$such that 
(6.1) $\left|\varphi^{(\mu, m)}-\varphi_{h}^{(\mu, m)}\right| \leq \alpha_{0}(h) \quad$ on $E_{0 . h} \cup \partial_{0} E_{h} \quad$ and $\quad \lim _{h \rightarrow 0} \alpha_{0}(h)=0$.

Then there is an $\alpha: H \rightarrow \mathbb{R}_{+}$such that

$$
\|r\|_{h}(\mu) \leq \alpha(h) \quad \text { for } 0 \leq \mu \leq K_{0} \quad \text { and } \quad \lim _{h \rightarrow 0} \alpha(h)=0 .
$$

Proof. We apply Theorem 5.1. Consider the operator $F_{h}: E_{h}^{+} \times F\left(\Omega_{h}, \mathbb{R}\right)$ $\rightarrow \mathbb{R}$ defined by

$$
F_{h}[a]^{(\mu, m)}:=a^{(\mu, m)}+h_{0} f\left(t^{(\mu)}, x^{(m)}, S_{h}[a], \delta a^{(\mu, m)}, \delta^{(2)} a^{(\mu, m)}\right) .
$$

Then $v$ satisfies (5.1), (5.2) and there is a function $\gamma: H \rightarrow \mathbb{R}_{+}$such that

$$
\left|U^{(\mu+1, m)}-F_{h}[U]^{(\mu, m)}\right| \leq h_{0} \gamma(h) \quad \text { on } E_{h}^{+}
$$

and $\lim _{h \rightarrow 0} \gamma(h)=0$. Let a constant $d \geq 0$ be such that

$$
\left|D_{i j} u(t, x)\right| \leq d \quad \text { for }(t, x) \in \Omega, i, j=1, \ldots, n
$$

$\left(\right.$ see $\left.\left(F_{5}\right)\right)$. We denote by $Y_{h}$ the class of all functions $a \in F\left(\Omega_{h}, \mathbb{R}\right)$ with the property:

$$
\left|\delta_{i j} a^{(\mu, m)}\right| \leq 3 d \quad \text { for }\left(t^{(\mu)}, x^{(m)}\right) \in E_{h}^{+}, i, j=1, \ldots, n .
$$

Obviously, $U \in Y_{h}$. Suppose that $a \in F\left(\Omega_{h}, \mathbb{R}\right), \bar{a} \in Y_{h}$ and $\left(t^{(\mu)}, x^{(m)}\right) \in E_{h}^{+}$. We prove that

$$
\left|F_{h}[a]^{(\mu, m)}-F_{h}[\bar{a}]^{(\mu, m)}\right| \leq\|a-\bar{a}\|_{h}(\mu)+h_{0} \varrho(3 d) \sigma\left(t^{(\mu)},\|a-\bar{a}\|_{h}(\mu)\right) .
$$

It follows from Assumption $F[f, u]$ that

$$
+\left|(a-\bar{a})^{(\mu, m)}+h_{0} \sum_{i=1}^{n} \alpha_{i}(P) \delta_{i}(a-\bar{a})^{(\mu, m)}+h_{0} \sum_{i, j=1}^{n} \beta_{i j}(P) \delta_{i j}(a-\bar{a})^{(\mu, m)}\right|,
$$

where $P=\left(t^{(\mu)}, x^{(m)}, S_{h}[a], S_{h}[a], \delta[a]^{(\mu, m)}, \delta[\bar{a}]^{(\mu, m)}, \delta^{(2)}[a]^{(\mu, m)}, \delta^{(2)}[\bar{a}]^{(\mu, m)}\right)$ $\in \Delta_{1}$. Write

$$
\begin{aligned}
S^{(0)}(P) & =1-2 h_{0} \sum_{i=1}^{n} \frac{1}{h_{i}^{2}} \beta_{i i}(P)+h_{0} \sum_{(i, j) \in \Gamma} \frac{1}{h_{i} h_{j}}\left|\beta_{i j}(P)\right|, \\
S_{+}^{(i)}(P) & =\frac{h_{0}}{2 h_{i}} \alpha_{i}(P)+\frac{h_{0}}{h_{i}^{2}} \beta_{i i}(P)-h_{0} \sum_{\substack{j \neq i \\
j=1}}^{n} \frac{1}{h_{i} h_{j}}\left|\beta_{i j}(P)\right|, \\
S_{-}^{(i)}(P) & =-\frac{h_{0}}{2 h_{i}} \alpha_{i}(P)+\frac{h_{0}}{h_{i}^{2}} \beta_{i i}(P)-h_{0} \sum_{\substack{j \neq i \\
j=1}}^{n} \frac{1}{h_{i} h_{j}}\left|\beta_{i j}(P)\right|,
\end{aligned}
$$

where $i=1, \ldots, n$. After grouping the expressions in (6.6) appropriately, in view of assumption $\left(F_{4}\right)$, the definitions of the difference operators and the 
relation

$$
\left\|S_{h}[a-\bar{a}]\right\|\left(t^{(\mu)}\right)=\|a-\bar{a}\|_{h}(\mu) \quad \text { for } \mu=0, \ldots, K_{0},
$$

we get

$$
\begin{aligned}
& \left|F_{h}[a]^{(\mu, m)}-F_{h}[\bar{a}]^{(\mu, m)}\right| \\
\leq & h_{0} \varrho(3 d) \sigma\left(t^{(\mu)},\|a-\bar{a}\|_{h}(\mu)\right)+\left|S^{(0)}(P)(a-\bar{a})^{(\mu, m)}\right| \\
& +\left|\sum_{i=1}^{n} S_{+}^{(i)}(P)(a-\bar{a})^{\left(\mu, m+e_{i}\right)}\right|+\left|\sum_{i=1}^{n} S_{-}^{(i)}(P)(a-\bar{a})^{\left(\mu, m-e_{i}\right)}\right| \\
& +h_{0} \sum_{(i, j) \in \Gamma_{+}} \frac{1}{2 h_{i} h_{j}} \beta_{i j}(P)\left[\left|(a-\bar{a})^{\left(\mu, m+e_{i}+e_{j}\right)}\right|+\left|(a-\bar{a})^{\left(\mu, m-e_{i}-e_{j}\right)}\right|\right] \\
& -h_{0} \sum_{(i, j) \in \Gamma_{-}} \frac{1}{2 h_{i} h_{j}} \beta_{i j}(P)\left[\left|(a-\bar{a})^{\left(\mu, m+e_{i}-e_{j}\right)}\right|+\left|(a-\bar{a})^{\left(\mu, m-e_{i}+e_{j}\right)}\right|\right] .
\end{aligned}
$$

Note that assumptions $\left(F_{3}\right)$ and $\left(S_{1}\right)$ imply

$$
S^{(0)}(P) \geq 0, \quad S_{+}^{(i)}(P) \geq 0, \quad S_{-}^{(i)}(P) \geq 0 \quad \text { for } i=1, \ldots, n
$$

and

$$
\begin{aligned}
S^{(0)}(P)+\sum_{i=1}^{n} S_{+}^{(i)}(P) & +\sum_{i=1}^{n} S_{-}^{(i)}(P) \\
& +h_{0} \sum_{(i, j) \in \Gamma_{+}} \frac{1}{h_{i} h_{j}} \beta_{i j}(P)-h_{0} \sum_{(i, j) \in \Gamma_{-}} \frac{1}{h_{i} h_{j}} \beta_{i j}(P)=1 .
\end{aligned}
$$

The above relations and (6.8) give (6.5).

Denote by $\eta: I_{h} \rightarrow \mathbb{R}_{+}$the solution of the initial difference problem

$$
\left\{\begin{array}{l}
\eta^{(\mu+1)}=\eta^{(\mu)}+h_{0} \varrho(3 d) \sigma\left(t^{(\mu)}, \eta^{(\mu)}\right)+h_{0} \gamma(h), \quad \mu=0, \ldots, K_{0}-1, \\
\eta^{(0)}=\alpha_{0}(h)
\end{array}\right.
$$

It follows from Theorem 5.1 that

$$
\|U-v\|_{h}(\mu) \leq \eta^{(\mu)}, \quad \mu=0, \ldots, K_{0} .
$$

Consider the Cauchy problem

$$
\omega^{\prime}(t)=\varrho(3 d) \sigma(t, \omega(t))+\gamma(h), \quad \omega(0)=\alpha_{0}(h),
$$

and its maximum solution $\omega(\cdot, h):[0, T] \rightarrow \mathbb{R}_{+}$(see assumption $\left(F_{4}\right)$ ). It easily follows that

$$
\eta^{(\mu)} \leq \omega\left(t^{(\mu)}, h\right) \leq \omega(T, h) \quad \text { for } \mu=0, \ldots, K_{0}
$$

and $\lim _{h \rightarrow 0} \omega(t, h)=0$ uniformly on $[0, T]$. Put $\alpha(h):=\omega(T, h)$. The proof is complete. 
6.2. Quasi-linear equation. We are interested in the numerical approximation of a classical solution of problem (1.3), (1.2).

We need the following assumptions on the functions $F, \varphi, a_{i j}$ and regularity of a solution $u$ of (1.3), (1.2), as well as on the steps $h$ of the mesh $\Omega_{h}$.

Assumption $Q F[F, A, u]$

$\left(Q F_{1}\right) F$ and $a_{i j}, i, j=1, \ldots, n$, are continuous on $\Delta^{F}$ and $\Delta^{A}$, respectively.

$\left(Q F_{2}\right)$ There exists a function $\alpha=\left(\alpha_{1}, \ldots, \alpha_{n}\right)$ with $\alpha_{i}: \Delta_{1}^{F} \rightarrow \mathbb{R}$ such that for any $(t, x, z, p),(t, x, z, \bar{p}) \in \Delta^{F}$,

$$
F(t, x, z, p)-F(t, x, z, \bar{p})=\sum_{i=1}^{n} \alpha_{i}(P)\left(p_{i}-\bar{p}_{i}\right),
$$

where $P=(t, x, z, z, p, \bar{p}) \in \Delta_{1}^{F}$.

$\left(Q F_{3}\right) A=\left[a_{i j}\right]_{i, j=1}^{n}$ is symmetric.

$\left(Q F_{4}\right)$ There is a function $\sigma:[0, T] \times \mathbb{R}_{+} \rightarrow \mathbb{R}_{+}$such that:

(1) $\sigma$ is continuous and nondecreasing with respect to both variables; moreover, $\sigma(t, 0)=0$ for $t \in[0, T]$;

(2) for each $c \geq 1$ and $\varepsilon, \varepsilon_{0}>0$, the maximum solution of the Cauchy problem

$$
\omega^{\prime}(t)=c \sigma(t, \omega(t))+\varepsilon, \quad \omega(0)=\varepsilon_{0},
$$

is defined on $[0, T]$ and the function $\widetilde{\omega}(t)=0$ for $t \in[0, T]$ is the maximum solution of (6.14) for each $c \geq 1$ and $\varepsilon, \varepsilon_{0}=0$;

(3) the Perron type estimates

$$
\begin{aligned}
|F(t, x, z, p)-F(t, x, \bar{z}, p)| & \leq \sigma(t,\|z-\bar{z}\|(t)), \\
\left|a_{i j}(t, x, z)-a_{i j}(t, x, \bar{z})\right| & \leq \sigma(t,\|z-\bar{z}\|(t)),
\end{aligned}
$$

where $i, j=1, \ldots, n$, hold on $\Delta^{F}$ and $\Delta^{A}$, respectively. $\left(Q F_{5}\right) u \in C^{1,2}(\Omega, \mathbb{R})$ is a regular solution of $(1.3)$, (1.2).

\section{Assumption $Q S[h]$}

$\left(Q S_{1}\right)$ The steps $h=\left(h_{0}, h^{\prime}\right) \in H$ are such that

$$
\begin{array}{r}
1-2 h_{0} \sum_{i=1}^{n} \frac{1}{h_{i}^{2}} a_{i i}(t, x, z)+h_{0} \sum_{(i, j) \in \Gamma} \frac{1}{h_{i} h_{j}}\left|a_{i j}(t, x, z)\right| \geq 0, \\
-\frac{h_{i}}{2}\left|\alpha_{i}(P)\right|+a_{i i}(t, x, z)-h_{i} \sum_{\substack{j \neq i \\
j=1}}^{n} \frac{1}{h_{j}}\left|a_{i j}(t, x, z)\right| \geq 0
\end{array}
$$

for all $(t, x, z) \in \Delta^{A}$ and $P \in \Delta_{1}^{F}, i=1, \ldots, n$.

$\left(Q S_{2}\right)$ There is $c_{0}>0$ such that $h_{i} h_{j}^{-1} \leq c_{0}$ for $i, j=1, \ldots, n$. 
REMARK 6.1. Assumptions (1) and (3) in $\left(Q F_{4}\right)$ imply that the functional $F$ and coefficients $a_{i j}$ are of the Volterra type. Moreover, if $F$ is differentiable, then $\alpha$ in assumption $\left(Q F_{2}\right)$ involves derivatives of $F$.

We now put

$$
f(t, x, z, p, q):=\sum_{i, j=1}^{n} a_{i j}(t, x, z) q_{i j}+F(t, x, z, p)
$$

for $(t, x, z, p, q) \in \Delta$, and consider the difference method (4.1) with this $f$ for (1.3), (1.2). If we apply Theorem 6.1, then we need Assumptions $Q F[F, A, u]$, $Q S[h]$ and the following assumption on the matrix $A$ : for each $(i, j) \in \Gamma$, the function

$$
\widetilde{a}_{i j}(t, x, z):=\operatorname{sign} a_{i j}(t, x, z) \quad \text { for }(t, x, z) \in \Delta^{A}
$$

is constant $\left(\right.$ see $\left.\left(F_{3}\right)\right)$. It is easily seen that $\varrho(y):=n^{2} y+1$ for $y \in \mathbb{R}_{+}$ satisfies $\left(F_{4}\right)$.

We prove that the condition of the coefficients $a_{i j}$ being of the same sign in $\Delta^{A}$ can be omitted if we modify the difference operator $\delta^{(2)}$. More precisely, we consider problem (1.3), (1.2) with $\delta_{0}, \delta, \delta_{i i}, i=1, \ldots, n$, given in Section 2, and we define $\delta_{i j},(i, j) \in \Gamma$, by

$$
\begin{aligned}
& \delta_{i j} a^{(\mu, m)} \\
& \quad:= \begin{cases}\frac{1}{2}\left[\delta_{i}^{+} \delta_{j}^{-} a^{(\mu, m)}+\delta_{i}^{-} \delta_{j}^{+} a^{(\mu, m)}\right] & \text { if } a_{i j}\left(t^{(\mu)}, x^{(m)}, S_{h}[a]\right)<0, \\
\frac{1}{2}\left[\delta_{i}^{+} \delta_{j}^{+} a^{(\mu, m)}+\delta_{i}^{-} \delta_{j}^{-} a^{(\mu, m)}\right] & \text { if } a_{i j}\left(t^{(\mu)}, x^{(m)}, S_{h}[a]\right) \geq 0,\end{cases}
\end{aligned}
$$

where $a \in F\left(\Omega_{h}, \mathbb{R}\right),\left(t^{(\mu)}, x^{(m)}\right) \in E_{h}^{+}$. Observe that the finite difference functional scheme (4.1) with $f$ given by (6.19) and $\delta_{i j}$ by (6.20) depends on the sign of $a_{i j}$ at $\left(t^{(\mu)}, x^{(m)}, S_{h}[a]\right)$ and this sign does not have to be the same in $\Delta^{A}$.

Theorem 6.2. Let Assumptions $Q F[F, A, u]$ and $Q S[h]$ hold and suppose that there is a function $\alpha_{0}: H \rightarrow \mathbb{R}_{+}$such that

$$
\left|\varphi^{(\mu, m)}-\varphi_{h}^{(\mu, m)}\right| \leq \alpha_{0}(h) \quad \text { on } E_{0 . h} \cup \partial_{0} E_{h} \quad \text { and } \quad \lim _{h \rightarrow 0} \alpha_{0}(h)=0 .
$$

Then there is an $\alpha: H \rightarrow \mathbb{R}_{+}$such that

$$
\|r\|_{h}(\mu) \leq \alpha(h) \quad \text { for } 0 \leq \mu \leq K_{0} \quad \text { and } \quad \lim _{h \rightarrow 0} \alpha(h)=0 .
$$

Proof. The proof of this theorem is similar to that of Theorem 6.1. We apply Theorem 5.1. Consider the operator $F_{h}: E_{h}^{+} \times F\left(\Omega_{h}, \mathbb{R}\right) \rightarrow \mathbb{R}$ defined 
by

$$
\begin{aligned}
F_{h}[a]^{(\mu, m)}:= & a^{(\mu, m)}+h_{0} \sum_{i, j=1}^{n} a_{i j}\left(t^{(\mu)}, x^{(m)}, S_{h}[a]\right) \delta_{i j} a^{(\mu, m)} \\
& +h_{0} F\left(t^{(\mu)}, x^{(m)}, S_{h}[a], \delta a^{(\mu, m)}\right) .
\end{aligned}
$$

Then $v$ satisfies (5.1), (5.2) and there is a function $\gamma: H \rightarrow \mathbb{R}_{+}$such that

$$
\left|U^{(\mu+1, m)}-F_{h}[U]^{(\mu, m)}\right| \leq h_{0} \gamma(h) \quad \text { on } E_{h}^{+}
$$

and $\lim _{h \rightarrow 0} \gamma(h)=0$. Let a constant $d \geq 0$ be such that

$$
\left|D_{i j} u(t, x)\right| \leq d \quad \text { for }(t, x) \in \Omega, i, j=1, \ldots, n
$$

(see $\left.\left(Q F_{5}\right)\right)$. We denote by $Y_{h}$ the class of all functions $a \in F\left(\Omega_{h}, \mathbb{R}\right)$ with the property

$$
\left|\delta_{i j} a^{(\mu, m)}\right| \leq 3 d \quad \text { for }\left(t^{(\mu)}, x^{(m)}\right) \in E_{h}^{+}, i, j=1, \ldots, n .
$$

Obviously, $U \in Y_{h}$. Suppose that $a \in F\left(\Omega_{h}, \mathbb{R}\right), \bar{a} \in Y_{h}$ and $\left(t^{(\mu)}, x^{(m)}\right) \in$ $E_{h}^{+}$. We prove that

$$
\begin{aligned}
\mid F_{h}[a]^{(\mu, m)}- & F_{h}[\bar{a}]^{(\mu, m)} \mid \\
& \leq\|a-\bar{a}\|_{h}(\mu)+h_{0}\left(1+3 n^{2} d\right) \sigma\left(t^{(\mu)},\|a-\bar{a}\|_{h}(\mu)\right) .
\end{aligned}
$$

It follows from Assumption $Q F[F, A, u]$ that

$$
\begin{aligned}
\leq & h_{0}\left[1+\sum_{i, j=1}^{n}\left|\delta_{i j} \bar{a}^{(\mu, m)}\right|\right] \sigma\left(t^{(\mu)},\left\|S_{h}[a-\bar{a}]\right\|\left(t^{(\mu)}\right)\right) \\
& +\left|(a-\bar{a})^{(\mu, m)}+h_{0} \sum_{i=1}^{n} \alpha_{i}(P) \delta_{i}(a-\bar{a})^{(\mu, m)}+h_{0} \sum_{i, j=1}^{n} a_{i j}(Q) \delta_{i j}(a-\bar{a})^{(\mu, m)}\right|,
\end{aligned}
$$

where $P=\left(t^{(\mu)}, x^{(m)}, S_{h}[a], S_{h}[a], \delta[a]^{(\mu, m)}, \delta[\bar{a}]^{(\mu, m)}\right) \in \Delta_{1}^{F}, Q=\left(t^{(\mu)}\right.$, $\left.x^{(m)}, S_{h}[a]\right) \in \Delta^{A}$. Write

$$
\begin{aligned}
& S^{(0)}(Q)=1-2 h_{0} \sum_{i=1}^{n} \frac{1}{h_{i}^{2}} a_{i i}(Q)+h_{0} \sum_{(i, j) \in \Gamma} \frac{1}{h_{i} h_{j}}\left|a_{i j}(Q)\right|, \\
& S_{+}^{(i)}(P)=\frac{h_{0}}{2 h_{i}} \alpha_{i}(P)+\frac{h_{0}}{h_{i}^{2}} a_{i i}(Q)-h_{0} \sum_{\substack{j \neq i \\
j=1}}^{n} \frac{1}{h_{i} h_{j}}\left|a_{i j}(Q)\right|, \\
& S_{-}^{(i)}(P)=-\frac{h_{0}}{2 h_{i}} \alpha_{i}(P)+\frac{h_{0}}{h_{i}^{2}} a_{i i}(Q)-h_{0} \sum_{\substack{j \neq i \\
j=1}}^{n} \frac{1}{h_{i} h_{j}}\left|a_{i j}(Q)\right|,
\end{aligned}
$$


where $i=1, \ldots, n$. Let

$$
\Gamma_{+}^{(\mu, m)}:=\left\{(i, j) \in \Gamma: a_{i j}(Q) \geq 0\right\}, \quad \Gamma_{-}^{(\mu, m)}:=\Gamma \backslash \Gamma_{+}^{(\mu, m)} .
$$

Assumption $\left(Q F_{4}\right)$ and (6.26) lead to the estimate

$$
\begin{aligned}
\leq & h_{0}\left(1+3 n^{2} d\right) \sigma\left(t^{(\mu)},\|a-\bar{a}\|_{h}(\mu)\right)+\left|S^{(0)}(P)(a-\bar{a})^{(\mu, m)}\right| \\
& +\left|\sum_{i=1}^{n} S_{+}^{(i)}(P)(a-\bar{a})^{\left(\mu, m+e_{i}\right)}\right|+\left|\sum_{i=1}^{n} S_{-}^{(i)}(P)(a-\bar{a})^{\left(\mu, m-e_{i}\right)}\right| \\
& +h_{0} \sum_{(i, j) \in \Gamma_{+}^{(\mu, m)}} \frac{1}{2 h_{i} h_{j}} a_{i j}(Q)\left[\left|(a-\bar{a})^{\left(\mu, m+e_{i}+e_{j}\right)}\right|+\left|(a-\bar{a})^{\left(\mu, m-e_{i}-e_{j}\right)}\right|\right] \\
& -h_{0} \sum_{(i, j) \in \Gamma_{-}^{(\mu, m)}} \frac{1}{2 h_{i} h_{j}} a_{i j}(Q)\left[\left|(a-\bar{a})^{\left(\mu, m+e_{i}-e_{j}\right)}\right|+\left|(a-\bar{a})^{\left(\mu, m-e_{i}+e_{j}\right)}\right|\right] .
\end{aligned}
$$

Assumption $\left(Q S_{1}\right)$ and (6.27) imply (6.25).

An analysis similar to that in the proof of Theorem 6.1 shows that assertion $(6.22)$ is satisfied with $\alpha(h):=\omega(T, h)$, where $\omega(\cdot, h):[0, T] \rightarrow \mathbb{R}_{+}$is the maximum solution of the Cauchy problem (6.11) with $\varrho(y):=n^{2} y+1$, $y \in \mathbb{R}_{+}$. This concludes the proof.

REMARK 6.2. Suppose that the assumptions of Theorems 6.1 or 6.2 are satisfied and, moreover, there is a constant $\bar{c}>0$ such that

$$
\left|\delta^{(2)} w^{(\mu, m)}\right| \leq \bar{c} \quad \text { on } E_{h}^{+}
$$

for all solutions $w \in F\left(\Omega_{h}, \mathbb{R}\right)$ of the disturbed finite difference functional schemes of (4.1). It follows from an analysis of the proofs of these theorems that the difference methods presented are stable.

REMARK 6.3. It is easy to see that all the results of the paper can be extended to weakly coupled differential functional systems. One part of each system can be strongly nonlinear and the other quasi-linear. This is a new result even in the case of systems without functional terms.

REMARK 6.4. If we assume that

$$
\sigma(t, y):=L y, \quad(t, y) \in[0, T] \times \mathbb{R}_{+},
$$

then the comparison difference problem can be solved and the errors of the difference methods can be estimated; see Remark 5.1.

6.3. Approximation by the interpolation operator. Lemma 6.1 below shows that the step operator $S_{h}$ in the finite difference functional scheme (4.1) can be replaced by the interpolation operator $T_{h}$, and all of the above proofs remain in force (see $(6.7),(6.29)$ ). The errors of the methods with $T_{h}$ 
are smaller than with $S_{h}$ if the appropriate regularity of a classical solution $u$ of the differential functional problems (1.1), (1.2) or (1.3), (1.2) is assumed (see Remark 6.5 and Example 2). But the computation time is longer.

Lemma 6.1. If $a \in F\left(\Omega_{h}, \mathbb{R}\right)$, then

$$
\left\|T_{h}[a]\right\|\left(t^{(\mu)}\right)=\|a\|_{h}(\mu) \quad \text { for } \mu=0, \ldots, K_{0} .
$$

Proof. It is easy to prove by induction on $n$ that

$$
\sum_{s \in S_{+}}\left(\frac{x-x^{(m)}}{h^{\prime}}\right)^{s}\left(1-\frac{x-x^{(m)}}{h^{\prime}}\right)^{1-s}=1 \quad \text { for } x^{(m)} \leq x \leq x^{(m+1)}
$$

By (6.30), we get (6.29).

Lemma 6.2. Suppose that $u \in C^{1}(\Omega, \mathbb{R})$ and denote by $U \in F\left(\Omega_{h}, \mathbb{R}\right)$ the restriction of $u$ to the set $\Omega_{h}$, i.e., $U^{M}:=u\left(x^{M}\right)$. Let

$$
C:=\max _{i=1, \ldots, n}\left\{\left\|D_{t} u\right\|_{\Omega},\left\|D_{i} u\right\|_{\Omega}\right\} .
$$

Then

$$
\left\|S_{h}[U]-u\right\|_{\Omega} \leq C\|h\|_{\Sigma}, \quad\left\|T_{h}[U]-u\right\|_{\Omega} \leq C\|h\|_{\Sigma},
$$

where $\|h\|_{\Sigma}:=h_{0}+h_{1}+\cdots+h_{n}$.

Proof. This lemma can be proved with the use of the mean value theorem and the method applied in the proof of Theorem 5.27 in [9].

Let $D_{t t}:=\partial^{2} / \partial t^{2}, D_{t i}:=\partial^{2} / \partial x_{i} \partial t$ for $i=1, \ldots, n$, where $t \in \mathbb{R}$, $x=\left(x_{1}, \ldots, x_{n}\right) \in \mathbb{R}^{n}$.

Lemma 6.3. Suppose that $u \in C^{2}(\Omega, \mathbb{R})$ and denote by $U \in F\left(\Omega_{h}, \mathbb{R}\right)$ the restriction of $u$ to the set $\Omega_{h}$. Let

$$
C:=\max _{i, j=1, \ldots, n}\left\{\left\|D_{t t} u\right\|_{\Omega},\left\|D_{t i} u\right\|_{\Omega},\left\|D_{i j} u\right\|_{\Omega}\right\} .
$$

Then

$$
\left\|T_{h}[U]-u\right\|_{\Omega} \leq C\|h\|_{\Sigma}^{2}
$$

Proof. This is a consequence of Theorem 5.27 in [9].

Remark 6.5. Suppose that Assumptions $F[f, u], S[h]$ or Assumptions $Q F[F, A, u], Q S[h]$ are satisfied. It follows from (2.13), (6.20), Lemmas 6.2, 6.3 , the mean value theorem and the Taylor formula that if $u \in C^{2,3}(\Omega, \mathbb{R})$, then $r=O(\|h\|)$ for $S_{h}$ and $T_{h}$. But if $u \in C^{2,4}(\Omega, \mathbb{R})$, then $r=O\left(h_{0}+\left\|h^{\prime}\right\|^{2}\right)$ for $T_{h}$ and $r=O(\|h\|)$ for $S_{h}$.

7. Numerical results. To illustrate the class of problems which can be treated with our methods, we consider a strongly nonlinear differential equation with a quasi-linear term, two quasi-linear differential integral equations with deviated variables and a system of differential integral equations with 
deviated variables. One equation in the system is strongly nonlinear and the other is quasi-linear. Dirichlet's problems below cannot be solved with the numerical methods known to date.

Put $n=2$. Let $E=[0,1] \times(-1,1)^{2}, E_{0}=\{0\} \times[-1,1]^{2}$ and $\partial_{0} E=$ $[0,1] \times\left([-1,1]^{2} \backslash(-1,1)^{2}\right)$.

EXAMPLE 1. Consider the strongly nonlinear differential equation

$$
\begin{aligned}
D_{t} u(t, x, y)= & \arctan \left[D_{11} u(t, x, y)+D_{12} u(t, x, y)+D_{22} u(t, x, y)\right] \\
& +[1+\cos u(t, x, y)]\left[D_{11} u(t, x, y)+D_{12} u(t, x, y)\right. \\
& \left.+D_{22} u(t, x, y)\right]+g(t, x, y)
\end{aligned}
$$

for $(t, x, y) \in E$, with the initial-boundary condition

$$
u(t, x, y)=\sin t \cos (x+y) \quad \text { for }(t, x, y) \in E_{0} \cup \partial_{0} E,
$$

where $g(t, x, y)=\arctan [3 \sin t \cos (x+y)]+(3 \sin t+\cos t) \cos (x+y)+$ $3 \sin t \cos (x+y) \cos [\sin t \cos (x+y)]$.

Observe that the right-hand side of (7.1) has a strongly nonlinear term and a quasi-linear term. The function $u(t, x, y)=\sin t \cos (x+y)$ is an analytic solution of (7.1), (7.2). Put $h_{0}=10^{-5}, h_{1}=h_{2}=2 \cdot 10^{-2}$. Let $\varepsilon_{\max }, \varepsilon_{\text {mean }}$ be the largest and mean values, respectively, of the errors $|r|$ at time $t^{(\mu)}$.

Table 1. Errors of the difference method with $S_{h}$

\begin{tabular}{ccc}
\hline$t^{(\mu)}$ & $\varepsilon_{\max }$ & $\varepsilon_{\text {mean }}$ \\
\hline 0.1 & $3.30 \cdot 10^{-6}$ & $1.62 \cdot 10^{-6}$ \\
0.2 & $9.68 \cdot 10^{-6}$ & $4.49 \cdot 10^{-6}$ \\
0.3 & $1.67 \cdot 10^{-5}$ & $7.62 \cdot 10^{-6}$ \\
0.4 & $2.40 \cdot 10^{-5}$ & $1.08 \cdot 10^{-5}$ \\
0.5 & $3.13 \cdot 10^{-5}$ & $1.40 \cdot 10^{-5}$ \\
0.6 & $3.88 \cdot 10^{-5}$ & $1.72 \cdot 10^{-5}$ \\
0.7 & $4.63 \cdot 10^{-5}$ & $2.04 \cdot 10^{-5}$ \\
0.8 & $5.40 \cdot 10^{-5}$ & $2.36 \cdot 10^{-5}$ \\
0.9 & $6.16 \cdot 10^{-5}$ & $2.68 \cdot 10^{-5}$ \\
1.0 & $6.91 \cdot 10^{-5}$ & $2.99 \cdot 10^{-5}$ \\
\hline & &
\end{tabular}

EXAMPLE 2. Consider the quasi-linear differential integral equation with deviated variables

$$
\begin{aligned}
D_{t} u(t, x, y)= & {\left[2+\cos \left(\int_{-x-y}^{x} \int_{-y}^{y} u(t, \xi, \zeta) d \zeta d \xi\right)\right]\left[D_{11} u(t, x, y)\right.} \\
& \left.+D_{12} u(t, x, y)+D_{22} u(t, x, y)\right]+\left|D_{1} u(t, x, y)\right| \\
& +u(0.5 t, 0,0)+g(t, x, y)
\end{aligned}
$$

for $(t, x, y) \in E$, with the initial-boundary condition

$$
u(t, x, y)=\sin t \cos (x+y) \quad \text { for }(t, x, y) \in E_{0} \cup \partial_{0} E,
$$

where $g(t, x, y)=(9 \sin t+\cos t) \cos (x+y)-\sin (0.5 t)-\sin t|\sin (x+y)|$. 
The function $u(t, x, y)=\sin t \cos (x+y)$ is an analytic solution of (7.3), (7.4). Put $h_{0}=10^{-5}, h_{1}=h_{2}=2 \cdot 10^{-2}$. Let $\varepsilon_{\max }, \varepsilon_{\text {mean }}$ be the largest and mean values, respectively, of the errors $|r|$ at time $t^{(\mu)}$.

Table 2. Errors of the difference method with $S_{h}$

\begin{tabular}{ccc}
\hline$t^{(\mu)}$ & $\varepsilon_{\max }$ & $\varepsilon_{\text {mean }}$ \\
\hline 0.1 & $3.86 \cdot 10^{-5}$ & $9.79 \cdot 10^{-6}$ \\
0.2 & $3.48 \cdot 10^{-4}$ & $9.62 \cdot 10^{-5}$ \\
0.3 & $1.22 \cdot 10^{-3}$ & $3.65 \cdot 10^{-4}$ \\
0.4 & $2.94 \cdot 10^{-3}$ & $9.23 \cdot 10^{-4}$ \\
0.5 & $5.73 \cdot 10^{-3}$ & $1.85 \cdot 10^{-3}$ \\
0.6 & $9.74 \cdot 10^{-3}$ & $3.23 \cdot 10^{-3}$ \\
0.7 & $1.50 \cdot 10^{-2}$ & $5.07 \cdot 10^{-3}$ \\
0.8 & $2.16 \cdot 10^{-2}$ & $7.37 \cdot 10^{-3}$ \\
0.9 & $2.92 \cdot 10^{-2}$ & $1.00 \cdot 10^{-2}$ \\
1.0 & $3.75 \cdot 10^{-2}$ & $1.30 \cdot 10^{-2}$ \\
\hline
\end{tabular}

Table 3. Errors of the difference method with $T_{h}$

\begin{tabular}{ccc}
\hline$t^{(\mu)}$ & $\varepsilon_{\max }$ & $\varepsilon_{\text {mean }}$ \\
\hline 0.1 & $3.24 \cdot 10^{-5}$ & $8.75 \cdot 10^{-6}$ \\
0.2 & $2.92 \cdot 10^{-4}$ & $8.42 \cdot 10^{-5}$ \\
0.3 & $1.03 \cdot 10^{-3}$ & $3.19 \cdot 10^{-4}$ \\
0.4 & $2.47 \cdot 10^{-3}$ & $8.05 \cdot 10^{-4}$ \\
0.5 & $4.79 \cdot 10^{-3}$ & $1.61 \cdot 10^{-3}$ \\
0.6 & $8.12 \cdot 10^{-3}$ & $2.81 \cdot 10^{-3}$ \\
0.7 & $1.25 \cdot 10^{-2}$ & $4.40 \cdot 10^{-3}$ \\
0.8 & $1.78 \cdot 10^{-2}$ & $6.37 \cdot 10^{-3}$ \\
0.9 & $2.40 \cdot 10^{-2}$ & $8.67 \cdot 10^{-3}$ \\
1.0 & $3.07 \cdot 10^{-2}$ & $1.12 \cdot 10^{-2}$ \\
\hline
\end{tabular}

EXAMPLE 3. Consider the quasi-linear differential integral equation with deviated variables

$$
\begin{aligned}
D_{t} u(t, x, y)= & D_{11} u(t, x, y)+D_{22} u(t, x, y) \\
& +\left[\cos \left(\int_{-x-y}^{x} \int_{y}^{y} u(t, \xi, \zeta) d \zeta d \xi\right)\right] D_{12} u(t, x, y) \\
& +\left|D_{1} u(t, x, y)\right|+u(0.5 t, 0,0)+g(t, x, y)
\end{aligned}
$$

for $(t, x, y) \in E$, with the initial-boundary condition

$$
u(t, x, y)=\sin t \cos (x+y) \quad \text { for }(t, x, y) \in E_{0} \cup \partial_{0} E,
$$

where $g(t, x, y)=(3 \sin t+\cos t) \cos (x+y)-\sin (0.5 t)-\sin t|\sin (x+y)|$.

The function $u(t, x, y)=\sin t \cos (x+y)$ is an analytic solution of (7.5), (7.6). Put $h_{0}=10^{-5}, h_{1}=h_{2}=2 \cdot 10^{-2}$. Let $\varepsilon_{\max }, \varepsilon_{\text {mean }}$ be the largest and mean values, respectively, of the errors $|r|$ at time $t^{(\mu)}$. 
Table 4. Errors of the difference method with $T_{h}$

\begin{tabular}{ccc}
\hline$t^{(\mu)}$ & $\varepsilon_{\max }$ & $\varepsilon_{\text {mean }}$ \\
\hline 0.1 & $2.15 \cdot 10^{-5}$ & $4.33 \cdot 10^{-6}$ \\
0.2 & $2.21 \cdot 10^{-4}$ & $4.78 \cdot 10^{-5}$ \\
0.3 & $8.19 \cdot 10^{-4}$ & $1.94 \cdot 10^{-4}$ \\
0.4 & $2.00 \cdot 10^{-3}$ & $5.16 \cdot 10^{-4}$ \\
0.5 & $3.90 \cdot 10^{-3}$ & $1.07 \cdot 10^{-3}$ \\
0.6 & $6.58 \cdot 10^{-3}$ & $1.91 \cdot 10^{-3}$ \\
0.7 & $9.98 \cdot 10^{-3}$ & $3.06 \cdot 10^{-3}$ \\
0.8 & $1.40 \cdot 10^{-2}$ & $4.49 \cdot 10^{-3}$ \\
0.9 & $1.85 \cdot 10^{-2}$ & $6.18 \cdot 10^{-3}$ \\
1.0 & $2.32 \cdot 10^{-2}$ & $8.05 \cdot 10^{-3}$ \\
\hline
\end{tabular}

Put $n=1$. Let $E=[0,1] \times(-1,1), E_{0}=\{0\} \times[-1,1]$ and $\partial_{0} E=$ $[0,1] \times([-1,1] \backslash(-1,1))$.

EXAMPLE 4. Consider the system of differential integral equations with deviated variables

$$
\left\{\begin{array}{l}
D_{t} u(t, x)=\arctan \left[D_{11} u(t, x)\right]+\int_{0}^{t} v(\tau, x) d \tau+g_{1}(t, x), \\
D_{t} v(t, x)=[1+\cos (u(0.5 t, x))] D_{11} v(t, x)+u(t, x)+g_{2}(t, x)
\end{array}\right.
$$

for $(t, x) \in E$, with the initial-boundary condition

$$
u(t, x)=\sin t \cos x, \quad v(t, x)=\sin t \sin x \quad \text { for }(t, x) \in E_{0} \cup \partial_{0} E,
$$

where $g_{1}(t, x)=\arctan (\sin t \cos x)+\cos t(\cos x+\sin x)-\sin x, g_{2}(t, x)=$ $\cos (\sin (0.5 t) \cos x) \sin t \sin x+\sin x(\sin t+\cos t)-\sin t \cos x$.

The pair of functions $u(t, x)=\sin t \cos x, v(t, x)=\sin t \sin x$ is an analytic solution of (7.7), (7.8). Put $h_{0}=10^{-5}, h_{1}=h_{2}=2 \cdot 10^{-2}$. Let $\varepsilon_{\max }^{1}$, $\varepsilon_{\max }^{2}$ be the largest and $\varepsilon_{\text {mean }}^{1}, \varepsilon_{\text {mean }}^{2}$ mean values, respectively, of the errors $\left|r_{1}\right|,\left|r_{2}\right|$ at time $t^{(\mu)}$. The error $r_{1}$ is connected with $u$, and $r_{2}$ with $v$.

Table 5. Errors of the difference method with $S_{h}$

\begin{tabular}{ccccc}
\hline$t^{(\mu)}$ & $\varepsilon_{\max }^{1}$ & $\varepsilon_{\text {mean }}^{1}$ & $\varepsilon_{\max }^{2}$ & $\varepsilon_{\text {mean }}^{2}$ \\
\hline 0.1 & $1.85 \cdot 10^{-7}$ & $1.34 \cdot 10^{-7}$ & $1.26 \cdot 10^{-7}$ & $7.83 \cdot 10^{-8}$ \\
0.2 & $6.95 \cdot 10^{-7}$ & $4.86 \cdot 10^{-7}$ & $3.47 \cdot 10^{-7}$ & $2.07 \cdot 10^{-7}$ \\
0.3 & $1.44 \cdot 10^{-6}$ & $9.91 \cdot 10^{-7}$ & $6.02 \cdot 10^{-7}$ & $3.43 \cdot 10^{-7}$ \\
0.4 & $2.36 \cdot 10^{-6}$ & $1.60 \cdot 10^{-6}$ & $8.80 \cdot 10^{-7}$ & $4.82 \cdot 10^{-7}$ \\
0.5 & $3.38 \cdot 10^{-6}$ & $2.27 \cdot 10^{-6}$ & $1.17 \cdot 10^{-6}$ & $6.24 \cdot 10^{-7}$ \\
0.6 & $4.46 \cdot 10^{-6}$ & $2.99 \cdot 10^{-6}$ & $1.48 \cdot 10^{-6}$ & $7.67 \cdot 10^{-7}$ \\
0.7 & $5.58 \cdot 10^{-6}$ & $3.72 \cdot 10^{-6}$ & $1.80 \cdot 10^{-6}$ & $9.11 \cdot 10^{-7}$ \\
0.8 & $6.71 \cdot 10^{-6}$ & $4.46 \cdot 10^{-6}$ & $2.11 \cdot 10^{-6}$ & $1.05 \cdot 10^{-6}$ \\
0.9 & $7.83 \cdot 10^{-6}$ & $5.20 \cdot 10^{-6}$ & $2.43 \cdot 10^{-6}$ & $1.19 \cdot 10^{-6}$ \\
1.0 & $8.94 \cdot 10^{-6}$ & $5.92 \cdot 10^{-6}$ & $2.74 \cdot 10^{-6}$ & $1.33 \cdot 10^{-6}$ \\
\hline & & & &
\end{tabular}


The results shown in the tables are consistent with our mathematical analysis. The tables of errors are typical of difference methods. The computation was performed on a $\mathrm{PC}$ computer.

Acknowledgements. The author is grateful to the reviewer for his valuable remarks, which improved the entire paper.

\section{References}

[1] P. Besala and G. Paszek, Differential-functional inequalities of parabolic type in unbounded regions, Ann. Polon. Math. 38 (1980), 217-228.

[2] - - - On the uniqueness of solutions to a parabolic system of differential-functional equations, Demonstratio Math. 18 (1985), 1-16.

[3] S. Brzychczy, Existence and uniqueness of solutions of infinite systems of semilinear parabolic differential-functional equations in arbitrary domains in ordered Banach spaces, Math. Comput. Modelling 36 (2002), 1183-1192.

[4] -, Monotone iterative methods for infinite systems of reaction-diffusion-convection equations with functional dependence, Opuscula Math. 25 (2005), 29-99.

[5] R. Ciarski, Numerical approximations of parabolic differential functional equations with the initial boundary conditions of the Neumann type, Ann. Polon. Math. 84 (2004), 103-119.

[6] - Stability of difference equations generated by parabolic differential functional equations, Demonstratio Math. 38 (2005), 101-117.

[7] J. Hale and L. Verdyun, Introduction to Functional Differential Equations, Springer, 1993.

[8] Z. Kamont and H. Leszczyński, Stability of difference equations generated by parabolic differential-functional problems, Rend. Mat. Appl. (7) 16 (1996), 265-287.

[9] Z. Kamont, Hyperbolic Functional Differential Inequalities and Applications, Kluwer, Dordrecht, 1999.

[10] - Numerical approximations of difference functional equations and applications, Opuscula Math. 25 (2005), 109-130.

[11] Z. Kowalski, A difference method for a non-linear system of elliptic equations with mixed derivatives, Ann. Polon. Math. 38 (1980), 229-243.

[12] M. Malec, Schéma des différences finies pour un système d'équations paraboliques non linéaires avec dérivées mixtes, ibid. 34 (1977), 125-134.

[13] —, Sur une méthode des différences finies pour une équation non linéaire différentielle fonctionnelle aux dérivées mixtes, ibid. 36 (1979), 1-10.

[14] M. Malec, Cz. Mączka and W. Voigt, Weak difference-functional inequalities and their application to the difference analogue of non-linear parabolic differential-functional equations, Numer. Math. 11 (1983), 69-79.

[15] M. Malec and M. Rosati, A convergent scheme for non-linear systems of differential functional equations of parabolic type, Rend. Mat. Appl. (7) 3 (1983), 211-227.

[16] M. Malec and L. Sapa, A finite difference method for nonlinear parabolic-elliptic systems of second order partial differential equations, Opuscula Math. 27 (2007), 259-289.

[17] R. Mosurski, Difference method for an elliptic system of non-linear differentialfunctional equations, Univ. Iagel. Acta Math. 43 (2005), 181-199.

[18] C. V. Pao, Coupled nonlinear parabolic systems with time delays, J. Math. Anal. Appl. 196 (1995), 237-265. 
[19] C. V. Pao, Systems of parabolic equations with continuous and discrete delays, ibid. 205 (1997), 157-185.

[20] - Finite difference reaction-diffusion systems with coupled boundary conditions and time delays, ibid. 272 (2002), 407-434.

[21] R. Redheffer and W. Walter, Comparison theorems for parabolic functional inequalities, Pacific J. Math. 85 (1979), 447-470.

[22] J. Szarski, Differential Inequalities, Monograf. Mat. 43, PWN-Polish Sci. Publ., Warszawa, 1967.

[23] - Uniqueness of solutions of a mixed problem for parabolic differential-functional equations, Ann. Polon. Math. 28 (1973), 57-65.

[24] - Strong maximum principle for non-linear parabolic differential-functional inequalities in arbitrary domains, ibid. 31 (1975), 197-203.

[25] W. Walter, Differential and Integral Inequalities, Monograph, Springer, Berlin, 1970.

[26] J. Wu, Theory and Applications of Partial Functional Differential Equations, Springer, 1996.

Faculty of Applied Mathematics

AGH University of Science and Technology

Al. Mickiewicza 30

30-059 Kraków, Poland

E-mail: lusapa@mat.agh.edu.pl

Received 29.3.2007

and in final form 21.11.2007 